\title{
AVALIAÇÃO DO TRATAMENTO CIRÚRGICO DAS FRATURAS EM DUAS OU TRÊS PARTES DO ÚMERO PROXIMAL COM O "SISTEMA PARAQUEDAS"
}

\author{
EVALUATION OF SURGICAL TREATMENT OF FRACTURES IN TWO OR THREE PARTS OF THE \\ PROXIMAL HUMERUS WITH THE "PARACHUTE TECHNIQUE"
}

Antonio Carlos Tenor Junior', Fabiano Rebouças Ribeiro', Rômulo Brasil Filho', Cantidio Salvador Filardi Filho², Guilherme Leme da Costa $^{3}$, Eduardo Libório Menniti ${ }^{4}$

\section{RESUMO}

Objetivo: Avaliar, através dos resultados clínicos, a eficácia e a segurança da técnica cirúrgica denominada "sistema paraquedas" aplicada em pacientes adultos que sofreram fraturas desviadas e instáveis em duas ou em três partes do úmero proximal. Métodos: Durante o período de janeiro de 1995 a junho de 2006, 59 pacientes adultos com fraturas em duas ou em três partes desviadas e instáveis foram operados pelo Grupo de Ombro e Cotovelo do Serviço de Ortopedia e Traumatologia do Hospital do Servidor Público Estadual de São Paulo com a técnica do "sistema paraquedas”. Este método consiste em uma banda de tensão intramedular e fixação extra medular em “8” unindo os fragmentos da fratura, utilizando um parafuso esponjoso de $6,5 \mathrm{~mm}$ com rosca parcial, arruela e dois fios inabsorvíveis, produzindo uma síntese estável, com mínima agressão às partes moles vizinhas, não requerendo a posterior retirada do material. A forma final desta síntese lembra a figura de um paraquedas aberto. Os pacientes tiveram um seguimento pós-operatório mínimo de seis meses. Para o diagnóstico foram realizadas radiografias nas incidências da série trauma de ombro. As fraturas foram classificadas de acordo com o sistema proposto por Neer. Utilizamos a escala da Universidade da Califórnia em Los Angeles (UCLA) na avaliação dos resultados. Resultados: Obteve-se $47 \%$ de bons e $26 \%$ de excelentes resultados com a técnica do "sistema paraquedas" de acordo com o escore da UCLA. Conclusão: A técnica do "sistema paraquedas” é opção segura e eficaz de tratamento das fraturas desviadas e instáveis em duas ou três partes do úmero proximal.

Descritores - Ombro; Fraturas do úmero/cirurgia; Osteossíntese

\section{ABSTRACT}

Objective: To evaluate, through clinical outcomes, the efficacy and safety of the surgical technique called the "parachute technique" as applied to adult patients who suffered displaced and unstable fractures in two or three parts of the proximal humerus. Methods: During the period from January 1995 to June 2006, 59 adult patients with two or three displaced and unstable fractures were operated by the Shoulder and Elbow Group of the Orthopedics and Traumatology Service of the Servidor Público Estadual de São Paulo's Hospital using the "parachute technique". This method consists of an internal bone tension band and a settling external bone in " 8 " joining the pieces of the fractures, using a 6.5-millimeter spongy screw with a partial thread, a washer and two nonabsorbable sutures, creating a stable synthesis with little aggression to the surrounding soft tissue, requiring no posterior material ressection. The final shape of this synthesis is reminiscent of the shape of an opened parachute. The patients had a minimum of 6 months follow-up post-operation. For diagnosis, trauma series radiographs of the shoulder were taken. The fractures were classified according to the system proposed by Neer. We used the scale of the University of California, Los Angeles (UCLA), in the evaluation of results. Results: There were $47 \%$ good and $26 \%$ excellent results using the "parachute technique" according to the UCLA score. Conclusion: the "parachute technique" is a safe and effective treatment for two or three part displaced and unstable fractures of the proximal humerus.

Keywords - Shoulder; Humeral fractures/ surgery; Osteosynthesis

\footnotetext{
1 - Médico Assistente do Grupo de Ombro do Serviço de Ortopedia e Traumatologia do HSPE - IAMSPE - São Paulo.

2 - Chefe do Grupo de Ombro do Serviço de Ortopedia e Traumatologia do HSPE - IAMSPE - São Paulo.

3 - Estagiário do Grupo de Ombro do Serviço de Ortopedia e Traumatologia do HSPE - IAMSPE - São Paulo.

4 - Médico Colaborador do Grupo de Ombro do Serviço de Ortopedia e Traumatologia do HSPE - IAMSPE - São Paulo.

Trabalho realizado no Departamento de Ortopedia e Traumatologia do Hospital do Servidor Público Estadual Francisco Morato de Oliveira - IAMSPE.

Correspondência: Rua Francisco Leitão, 474, ap. 92, Pinheiros - São Paulo, SP - E-mail: actenorjr@hotmail.com
} 


\section{INTRODUÇÃO}

A incidência das fraturas do úmero proximal é estimada em torno de $3 \%$ a $5 \%$ de todas as fraturas, sendo maior após os 50 anos, com aproximadamente $80 \%$ dos casos no sexo feminino. É a terceira maior causa de fraturas em pacientes idosos ${ }^{(1-4)}$.

Codman descreveu as quatro partes anatômicas básicas do úmero proximal: cabeça, tubérculo maior, tubérculo menor e diáfise, levando em consideração a sua vascularização. A classificação mais utilizada para as fraturas do úmero proximal foi descrita por Neer, baseada no desvio entre os quatro fragmentos descritos por Codman, com os objetivos de estabelecer o prognóstico da viabilidade da cabeça do úmero e orientar a conduta ${ }^{(5)}$.

O tratamento conservador está indicado em até 85\% dos casos, decorrentes de traumas de baixa energia, por apresentarem pouco desvio entre os fragmentos (até $1 \mathrm{~cm} \mathrm{ou} 45^{\circ}$, segundo os critérios de Neer), ficando reservado o tratamento cirúrgico para as fraturas desviadas, instáveis ou com lesões vasculonervosas associadas $^{(1-3)}$. As fraturas com desvio em duas e em três partes e as fraturas-luxações apresentam resultados insatisfatórios quando submetidas ao tratamento conservador, devido à dificuldade em se obter a redução incruenta, por interposição das partes moles adjacentes e por ação da musculatura da cintura escapular ${ }^{(1,2,6)}$.

Há grande número de técnicas cirúrgicas descritas para o tratamento das fraturas do úmero proximal, com opiniões diversas quanto ao melhor método de fixação, sobretudo para pacientes idosos, com osteoporose ${ }^{(1)}$. As técnicas mais utilizadas são as osteossínteses rígidas, com placas e parafusos, e as bandas de tensão, associadas ou não a hastes intramedulares flexíveis ${ }^{(2)}$. Há também técnicas minimamente invasivas, como a redução fechada e fixação percutânea ${ }^{(3)}$. As técnicas que utilizam placas e parafusos conferem maior estabilidade em ossos de boa qualidade, porém suas eficácias diminuem em ossos poróticos ${ }^{(1)}$.

O objetivo deste trabalho é avaliar, clínica e radiograficamente, os resultados da técnica que associa uma banda de tensão intramedular a outra banda de tensão extra medular em "8”, com parafuso, arruela e fios inabsorvíveis, denominada "sistema paraquedas", descrita e publicada previamente na Revista Brasileira de Ortopedia, em setembro de $1997^{(2)}$.

\section{MÉTODOS}

No período de janeiro de 1995 a junho de 2006, 59 ombros de 59 pacientes adultos foram operados pelo Grupo de Ombro e Cotovelo do Serviço de Ortopedia e Traumatologia do Hospital do Servidor Público Estadual de São Paulo, para o tratamento de fraturas do úmero proximal em duas ou em três partes, segundo os critérios de Neer, através do "sistema paraquedas".

Foram excluídos do estudo sete pacientes, por apresentarem seguimento inferior a seis meses, e 26 pacientes por perderem o seguimento ambulatorial. A idade dos pacientes variou de 40 a 87 anos (média de 54 anos). O sexo feminino (72\%) predominou sobre o masculino (28\%) e o membro dominante foi operado em $72 \%$ dos casos. Os pacientes foram seguidos por tempo que variou de seis a 120 meses (média de 31 meses). O intervalo decorrido entre o trauma e tratamento cirúrgico variou de um a 21 dias (média de cinco dias). A queda sobre o solo foi o mecanismo de trauma responsável por $67 \%$ das fraturas (40 pacientes). Em 19 pacientes as fraturas ocorreram por traumas de alta energia (quatro atropelamentos, 12 acidentes automobilísticos, três acidentes motociclísticos).

A técnica consiste em uma banda de tensão intramedular obtida através de dois fios de Ethibond ${ }^{\circledR}$ número 5 , que circundam o seguimento liso da haste do parafuso esponjoso de 6,5mm (Figura 1), com rosca parcial e com arruela, colocado na diáfise umeral, distal ao foco de fratura, perpendicular ao sulco intertubercular. Após bloqueio do plexo braquial associado a anestesia geral, o paciente é posicionado em "cadeira de praia". É realizada uma via deltopeitoral clássica (Figuras 2a e b)

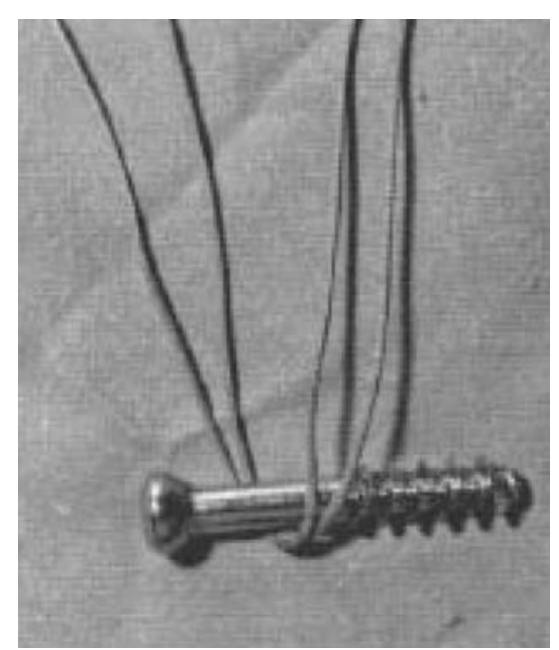

Figura 1 - Os fios circundam a região não rosqueada do parafuso 


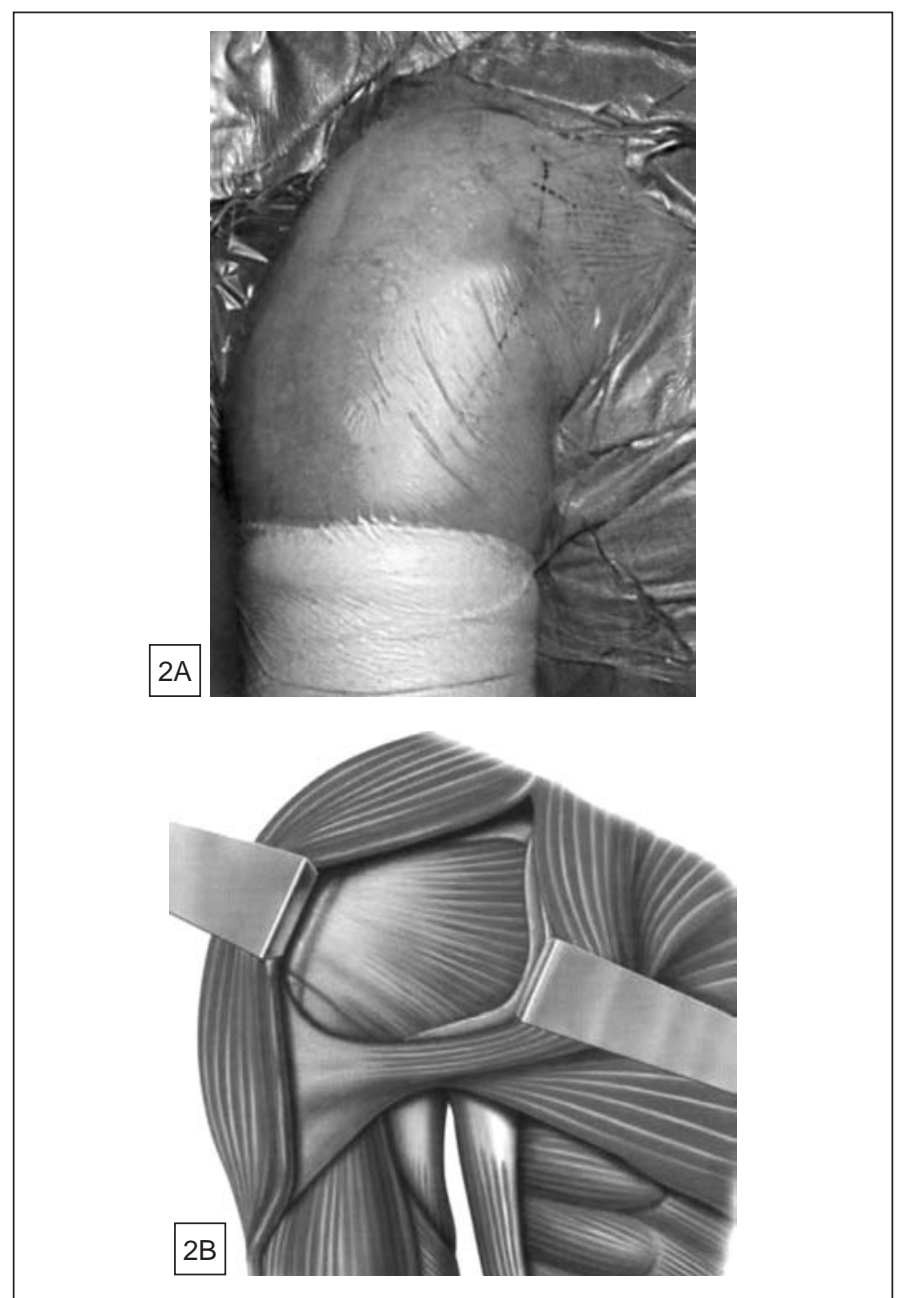

Figura 2 - A, B) Via de acesso deltopeitoral clássica

com exposição do foco da fratura. O primeiro fio de Ethibond ${ }^{\circledR}$ transfixa o tubérculo maior de fora para dentro, saindo na parte esponjosa deste fragmento. A seguir, posterior ao cabo longo do músculo bíceps braquial, o fio é reparado no seu ponto médio, formando uma alça. O tubérculo menor é transfixado, de dentro para fora, exteriorizando-se através do manguito rotador. Este procedimento é repetido com o segundo fio de Ethibond ${ }^{\circledR}$ e os fios devem ficar paralelos e equidistantes entre si. O canal medular umeral é isolado e as duas alças de Ethibond ${ }^{\circledR}$ reparadas são inseridas. Em seguida, o parafuso esponjoso de $6,5 \mathrm{~mm}$ com rosca parcial e com arruela é transfixado na diáfise umeral, de lateral para medial, perpendicularmente ao sulco intertubercular, fazendo com que as duas alças dos fios circundem a porção lisa (sem roscas) da haste do parafuso, de modo a ancorá-los.

Deste modo, os fios seguem trajeto intramedular em direção divergente e ascendente para os tubérculos menor e maior, formando uma estrutura que lembra um paraquedas aberto. Com os dois fios de Ethibond ${ }^{\circledR}$ temos quatro extremidades: duas mediais, para o tubérculo menor, e duas laterais, para o tubérculo maior (Figura 3).

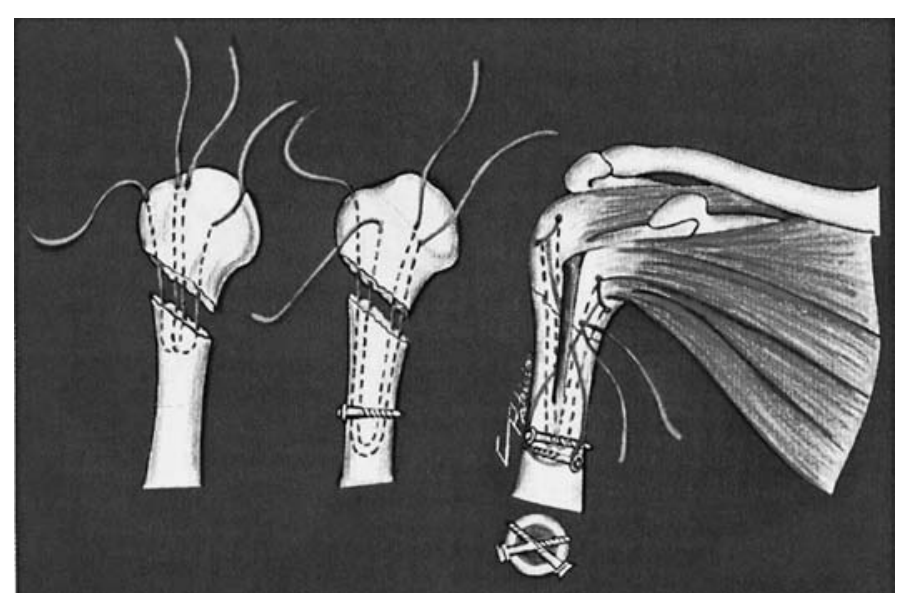

Figura 3 - "Sistema paraquedas", técnica que associa uma banda de tensão intramedular a outra banda de tensão extramedular em "8"

Em seguida, a fratura é reduzida e tencionam-se as quatro extremidades dos fios, amarrando inicialmente entre si as extremidades do tubérculo menor e, em seguida, as do tubérculo maior sobre o manguito rotador. Teremos, então, os dois grupos de fios duplos, um em cada tubérculo. Posteriormente, os fios são cruzados sob o tendão da cabeça longa do músculo bíceps braquial, tracionados e amarrados sob a arruela do parafuso previamente colocado na diáfise umeral, formando uma figura em “8” (Figuras 4a, b, c e d).

Consegue-se, assim, impacção e estabilização dos fragmentos através de dupla fixação, uma intramedular, à semelhança de um paraquedas aberto e outra extramedular, em "8” (Figuras 5a e b).

O protocolo pós-operatório é dividido em três fases. A primeira inicia-se no primeiro dia pós-operatório e consiste em um programa de movimentos ativos do cotovelo, do punho e da mão. A segunda fase, entre a terceira e a sexta semanas pós-operatórias, consiste em movimentos pendulares de Codman, elevação passiva de até $90^{\circ}$ e rotação passiva de até $20^{\circ}$. A terceira fase consiste em fisioterapia para ganho de amplitude de movimentos passivos e ativos, seguidos de um programa de exercícios para fortalecimento muscular, contra resistência, que começam entre a sexta e a oitava semanas pós-operatórias, quando obtida a consolidação clínica e radiográfica da fratura.

A avaliação clínica dos nossos resultados foi realizada por meio do sistema de pontos definidos pela Univer- 



Figura 4 - Técnica operatória: A) Transfixação do tubérculos com o fio, deixando uma alça; B) Inserção da alça do fio no canal medular e colocação do parafuso; C) Os fios são tensionados e a fratura é reduzida; D) Os fios são amarrados entre si e posteriormente sob a arruela do parafuso, formando uma figura em "8"
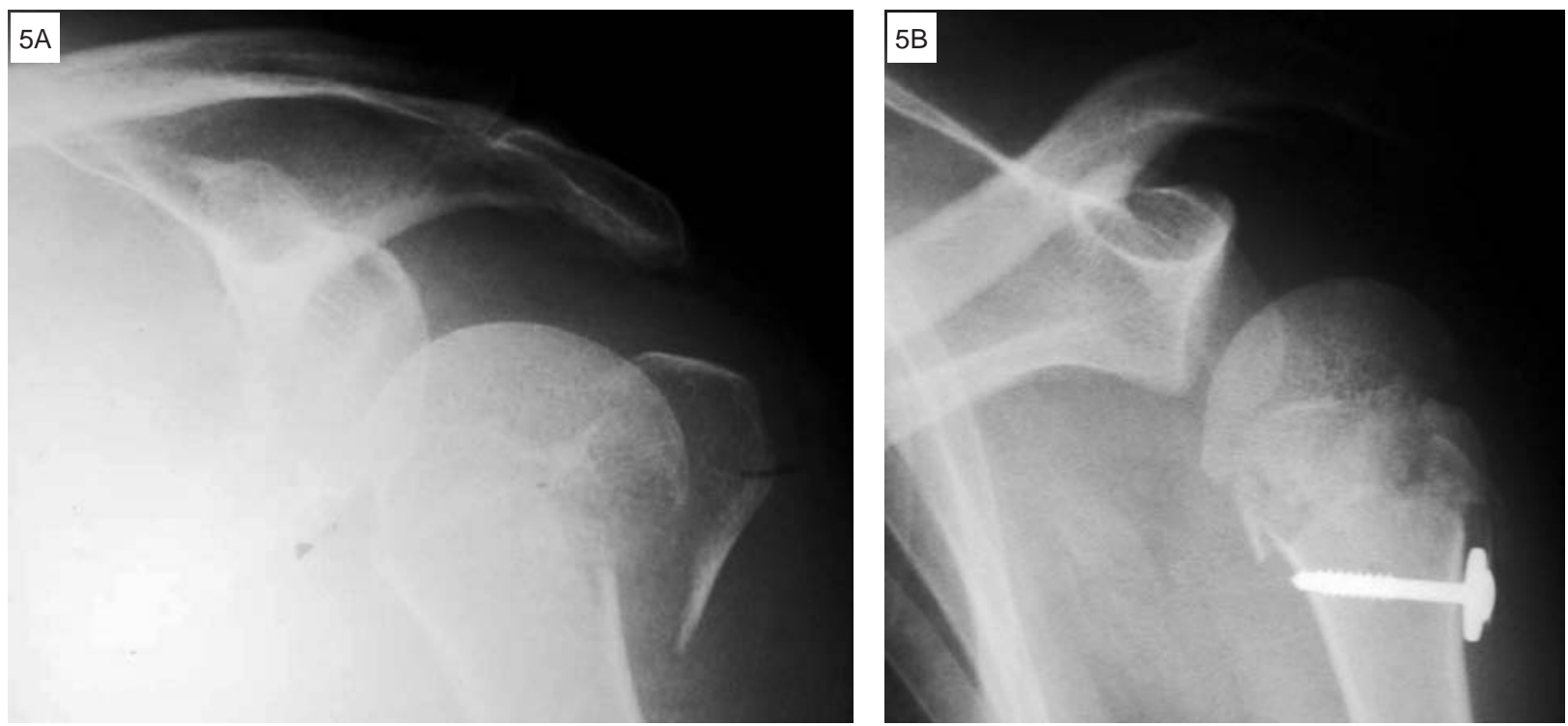

Figura 5 - A) Radiografia na posição anteroposterior mostrando fratura em três partes do úmero proximal. B) Radiografia na posição anteroposterior verdadeira do pós-operatório inicial desta mesma fratura utilizando a técnica "sistema paraquedas" 
sity of Califórnia at Los Angeles (UCLA) ${ }^{(7)}$ modificado por Ellman e $\mathrm{Kay}^{(7)}$, que se baseia em critérios objetivos e subjetivos, atribuindo pontos aos quesitos dor, grau de mobilidade, função do ombro, força e satisfação do paciente. A pontuação máxima é de 35 pontos. Para medir os graus de amplitude de movimento articular utilizamos o método descrito pela American Academy of Orthopaedic Surgeons.

\section{RESULTADOS}

Estavam disponíveis 59 pacientes para a avaliação da técnica do "sistema paraquedas". De acordo com o sistema de pontos da $\mathrm{UCLA}^{(7)}, 28$ pacientes (47\%) obtiveram 35 pontos e foram classificados como resultados excelentes; 15 pacientes (26\%), com 33 pontos, foram classificados como bons; nove pacientes (15\%), com 29 pontos, classificados como regulares; e sete pacientes (12\%), com 27 pontos, classificados como ruins.

A elevação ativa, depois de cumpridas as três etapas do protocolo de reabilitação, variou de $60^{\circ}$ a $180^{\circ}$ (média de $160^{\circ}$ ); a rotação lateral, de $5^{\circ}$ a $75^{\circ}$ (média de $\left.60^{\circ}\right)$ e a rotação medial, de polegar-glúteo a polegar-T8 (média de T12).

Complicações foram encontradas em seis pacientes: um com pseudoartrose, três com perda da redução, um com capsulite adesiva e um com infecção. Não houve nenhum caso de osteonecrose nas avaliações tardias dos pacientes.

\section{DISCUSSÃO}

A ação muscular sobre o úmero proximal, produzindo desvios, e a interposição de tecidos moles no foco de fratura, dificultam a obtenção e a manutenção da redução das fraturas desviadas. Nestes casos, a redução aberta e a fixação rígida, permitindo mobilização precoce, produzem melhores resultados que o tratamento incruento $^{(4,6)}$.

Não há consenso na literatura a respeito do melhor método de tratamento cirúrgico para as fraturas desviadas em duas ou em três partes do úmero proximal e a experiência com uma determinada técnica empregada por um grupo pode não ser reprodutível em outros centros. O agrupamento de diferentes tipos de fraturas em um mesmo trabalho, tanto pelas imperfeições dos sistemas de classificação quanto pela difícil reprodutibilidade intra e interobservadores, dificulta a comparação entre os métodos. A influência da idade do paciente no resultado do tratamento também é fator importante a ser considerado, pois nos idosos, além da porose, é frequente a lesão do manguito rotador. Porém, independente do método empregado, os melhores resultados são obtidos quando a redução com restauração da anatomia e a estabilidade são obtidas ${ }^{(4,8,9)}$.

A fixação percutânea das fraturas do úmero proximal apresenta a vantagem da mínima agressão aos tecidos moles vizinhos, porém não é isenta de complicações como lesão dos nervos axilar e radial. Apresenta ainda a desvantagem de conferir menor resistência aos desvios angulares $^{(4,6)}$.

O uso de bandas de tensão associadas a hastes flexíveis intramedulares confere boa estabilidade à síntese, inclusive em pacientes com ossos poróticos, com mínima agressão às partes moles vizinhas. No entanto, violam o manguito rotador e apresentam complicações como a migração proximal das hastes, produzindo síndrome do impacto subacromial, requerendo um segundo procedimento cirúrgico para a retirada do material de síntese $^{(6)}$.

A utilização de placas e parafusos permite boa fixação em ossos fortes, porém é frequente a soltura do material de síntese em ossos poróticos, devido à pega insuficiente dos parafusos, além da possibilidade de ocasionar síndrome do impacto subacromial por migração dos parafusos e necrose avascular por excessivas desperiostização e agressão aos tecidos moles vizinhos ${ }^{(1,4,5,8,10)}$.

Novas técnicas têm sido introduzidas com o objetivo de restabelecer a anatomia, como Plarus nail, Plan Tan Humerus Fixator Plate e Proximal humerus internal locked system, como a placa Philos. Não são isentas das mesmas complicações enumeradas acima, têm custo elevado e ainda há poucos estudos na literatura, com casuística e seguimento insuficientes para estudo comparativo entre os métodos de tratamento ${ }^{(4,10)}$. Desta nova geração de material de síntese, a placa Philos, de ângulo fixo, tem mostrado os melhores resultados em ossos frágeis, devido à melhor ancoragem dos parafusos do bloqueio ao osso ${ }^{(4,10)}$.

O “sistema paraquedas" tem se mostrado boa alternativa, na medida em que confere estabilidade à síntese, mesmo em ossos poróticos, aproveitando o manguito rotador como elemento auxiliar na estabilização dos fragmentos, com uso mínimo de material de síntese, baixo custo e mínima agressão às partes moles vizinhas, permitindo mobilização precoce e favorecendo a reabilitação do paciente (Figuras 6a, b, c, d). A impacção 

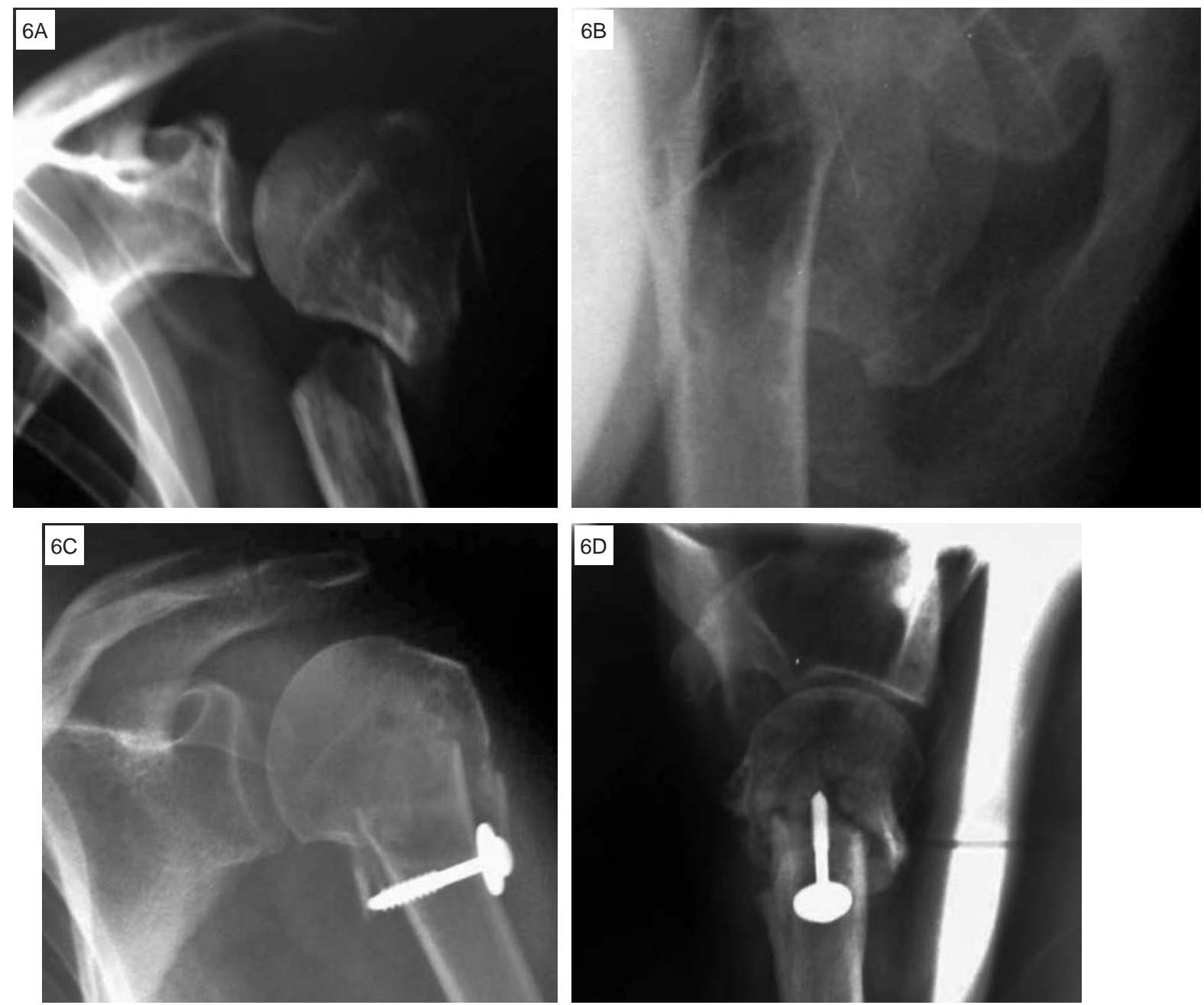

Figura 6 - Radiografias na posição anteroposterior verdadeira A) e axilar B) mostrando fratura em duas partes desviada do úmero proximal. Radiografias na posição anteroposterior verdadeira C) e axilar D) do pós-operatório inicial utilizando a técnica "sistema paraquedas"

obtida confere a estabilidade necessária, que é testada no intraoperatório. Não viola o manguito rotador e não interfere com a biomecânica do ombro. Não necessita de radioscopia, não provoca impacto subacromial e não requer a retirada do material de síntese ${ }^{(2)}$. Demonstra ser uma técnica reprodutível, com altos índices de excelentes e bons resultados e baixos índices de complicações após seguimento de 10 anos.

\section{REFERÊNCIAS}

1. Checchia SL, Doneux Santos P; Miyazaki NA, Fregoneze M, Silva LA, Lobo A et al. Avaliação do tratamento Cirúrgico da fratura em duas partes do colo cirúrgico do úmero com placa PFS ${ }^{\circledR}$ 80. Rer Bras Ortop. 2004;39(10):555-67.

2. Menniti EL, Brasil Filho Rômulo, Filardi Filho CS, Baptista MV, Daher SS. Banda de tensão intramedular com parafusos em fraturas do colo do úmero em duas e três partes: "sistema pára-quedas". Rev Bras Ortop. 1997;32(9):713-7.

3. Veado MAC, Silva NF, Meira MG. Redução fechada e pinagem percutânea das fraturas do úmero proximal. Rev Bras Ortop. 2002;37(4):122-8.

4. Helmy $N$, hintermann B. New trends in the treatment of proximal humerus fractures. Clin Orthop Relat Res. 2006;(422):100-8.

5. Checchia SL, Miranda DL, Carneiro UM, Cassani R. Tratamento das fraturas do colo cirúrgico do úmero pala técnica de Kapandjii. Rev Bras Ortop. 1993;28(1/2):43-9.

6. Ferreira Neto AA, Ferreira Filho AA, Zoppi Filho AA, Benegas E, Negri JH, Macha-

\section{CONCLUSÕES}

De acordo com os resultados obtidos, concluímos que o "sistema paraquedas" é uma técnica segura e eficaz para o tratamento cirúrgico das fraturas desviadas e instáveis em duas ou em três partes do úmero proximal, conferindo resultados bons e excelentes em $73 \%$ dos pacientes avaliados, o que nos permite considerá-la boa opção de tratamento.

do LFM, et al. Osteossíntese das fraturas em duas e Três partes da extremidade proximal do úmero com hastes de Ender modificadas com amarrilhos de "Ethibond". Rev Bras Ortop. 1997;32(7):707-12.

7. Ellman H, Kay SP. Arthroscopic subacromial decompression for chronic impingement. Two- to five-year results. J Bone Joint Surg Br. 1991;73(3):395-8.

8. Burton DJ, Wells G, Watters A, Schilders E, Venkateswaran B. Early experience with the PlantTan Fixator Plate for 2 and 3 part fractures of the proximal humerus. Injury. 2005;36(10):1190-6.

9. Hertel R. Fractures of the proximal humerus in osteoporotic bone. Osteoporos Int. 2005;16(Suppl 2):S65-72.

10. Koukakis A, Apostolou CD, Taneja T, Korres DS, Amini A. Fixation of proximal humerus fractures using the PHILOS plate: early experience. Clin Orthop Relat Res. 2006;(442):115-20. 